showed HIV positive. A positive CSF-VDRL test was shown in seven patients, three had HIV positive. 3) Peripheral blood $\mathrm{CD}^{+}{ }^{+} \mathrm{T}$ cell count The peripheral blood $\mathrm{CD} 4^{+} \mathrm{T}$ cell count was low $(<550$ cells $/ \mu \mathrm{L})$ in fifteen out of 26 malignant syphilis, of those seven cases were HIV seropositive. Six out of 26 patients suffered from both malignant syphilis and neurosyphilis but without HIV infection.

Conclusion There is no direct association between HIV infection and malignant syphilis or neurosyphilis. Additionally, we found a new unusual combination of malignant syphilis and neurosyphillis in the absence of HIV infection.

Disclosure No significant relationships.

\section{CCS01.2 A CASE SERIES OF SYPHILIS MASQUERADING AS THORACIC MALIGNANCY}

Jessica Doctor ${ }^{*}$, Achyuta Nori. Guys' and St Thomas's NHS Foundation Trust, London, UK

10.1136/sextrans-2019-sti.98

Background Infectious syphilis incidence is increasing worldwide, particularly amongst men who have sex with men (MSM). We report a case series of two patients with a rare presentation of syphilitic pneumonitis masquerading as thoracic malignancy.

Methods Patient history review via electronic records.

Results Case 1- 54 year old MSM, HIV diagnosed 2011, CD4 nadir 557 cells $/ \mu$, current CD4 861 cells/ $\mu$ l and HIV viral load (VL) undetectable since commencing antiretroviral therapy (ART), presented to emergency department (ED) with pleuritic chest pain and normal oxygen saturation. CT scan demonstrated parenchymal and sub-pleural nodules measuring maximum $13 \mathrm{~mm}$, thought likely to represent malignancy and one lesion had features of an infarct. He started Rivaroxaban in ED and subsequently developed a rash suggestive of evolving syphilis. Rapid plasma reagin (RPR) six weeks after initial presentation was 1:32. Treated with 2 weeks of doxycycline, RPR six weeks later was $1: 16$ and at five months 1:4. CT scan six weeks post treatment showed almost complete resolution of nodules. Case 2- 38 year old MSM, HIV diagnosed in March 2017, CD4 nadir 65 cells/ $\mu$, current CD4 219 cells/ $\mu \mathrm{l}$ and VL 109 cells/ $\mu$ l on ART. Kaposi's sarcoma (KS) diagnosed in 2017, involving skin, lymph nodes and spleen, treated to remission with ART alone. Presented in July 2018 with deranged liver function tests. CT scan showed multiple subpleural and parenchymal nodules, largest $11 \mathrm{~mm}$, however no progression of lymphadenopathy or clinical progression of cutaneous KS. RPR 1:512 and resolution to 1:16 three months after treatment with intramuscular Benzathine Penicillin. Radiological resolution of lung nodules occurred after 2 months of treatment. Chemotherapy was avoided.

Conclusion Syphilitic pulmonary/pleural nodules resolve with standard antibiotic therapy. Patients with pulmonary nodules usually present to primary care or specialist services where they are investigated for vasculitis/malignancy, but rarely for syphilis. For patients at risk, ruling out syphilis would avoid unnecessary interventions.

Disclosure No significant relationships.
CCSO1.3 SYPHILIS AS FIBROMYALGIA WITH UNEXPLAINED HEPATOSPLENOMEGALY

Jackie Sherrard*. Buckinghamshire Healthcare NHS Trust, Amersham, UK

10.1136/sextrans-2019-sti.99

Disclosure No significant relationships.

\section{CCSO2 - CLINICAL CASE SERIES - CLINICAL CASES FROM EXPERTS}

\section{Tuesday, July 16, 2019 7:00 AM - 8:00 AM}

\section{CCSO2.1 DEQUALINIUM CHLORIDE VAGINAL TABLETS FOR RECALCITRANT TRICHOMONAS VAGINALIS (TV): A CASE REPORT}

Deborah Goode*, Natasha Astill, Janet Wilson. Leeds Teaching Hospitals NHS Trust, Leeds Sexual Health, Leeds, UK

\subsection{6/sextrans-2019-sti.100}

Background Treatment for TV is often ineffective. Even very high-dose tinidazole has $8-10 \%$ failure and subsequent treatment options have limited evidence-base. Dequalinium has an EU license for bacterial vaginosis treatment. It is well tolerated, safe and has in-vitro activity against TV, but clinical experience is limited. We present the case of an 18-year old female with a 12-month history of persistent TV despite standard and resistant treatments, which finally responded to prolonged dequalinium.

Methods The patient was white British with no significant medical history. The presumed source of infection was a male living in Dubai. There was no risk of reinfection and adherence was self-reported as excellent throughout. Initial and subsequent presentations were with typical symptoms of vulvovaginitis and purulent vaginal discharge. Investigations were with onsite microscopy and TV nucleic acid amplification tests (NAAT). Treatment initially followed the British Association of Sexual Health and HIV TV Guideline. She received: several courses of 7-day and very high dose oral metronidazole (once with concurrent ampicillin and clotrimazole pessaries); intravenous metronidazole administered alongside vaginal metronidazole gel; oral tinidazole with intravaginal metronidazole. All nitroimidazole courses were up to 14 days duration. Vulvovaginitis symptoms settled during antimicrobial therapy, but recurred soon after cessation of treatment. At each follow-up TV was confirmed by microscopy and NAAT. We retreated with 4 -weeks of metronidazole $400 \mathrm{mg}$ twice daily with dequalinium intravaginal pessaries nightly. Symptoms were controlled, but TV NAAT and microscopy remained positive. As there was symptomatic relief from dequalinium, this was continued as monotherapy for a further 14 weeks pending sourcing alternative treatments.

Results Her symptoms remained controlled and microscopy and NAAT became negative. She remained asymptomatic with 